\title{
Estudio de Campo de una Vacuna B.C.G. Liofilizada
}

\author{
Q.F. Ivana Azzini B. ${ }^{1}$; Dra. Raquel Carraseo T. ${ }^{2}$; Dra. María de la livente $\mathrm{H}^{2}$ : \\ E.U. Ximena Ferrer S $G^{3}{ }^{3}$ : E. L. Cecilia Latrach $A^{3}{ }^{3}$ : Matr. Pilar Rius $F_{.}{ }^{4}$.
}

Field Study of a Freeze-Dried B.C.G. Vaccine

\begin{abstract}
Results from a ficld study perfomed on 116 infints 4 month old inoculatcd at birth with freeze-dricd BCG vaccinc produced by Rhodia Mericux Institute, France, are presented. This study was carticd out by the same investigators of a previous researcho done with freeze dricd BCG vaccine produced by Connaught Laboratories Canada, applying the same methods. The vaceine under study produced a scar similar to that of other strains previously rested in Chile. PPD response is small with a mean diameter of 4.5 mm $\pm 3.5 \mathrm{~mm}$ S.D. $\Lambda$ statistically significant difference was found $(p<0.001)$ when compated with the liquid BCG vaccine produced by the Public Health Institute of Chile. No differences were found between the freeze dried BCG vaccine produced by Rhodia Merieux and that from Connaught Laboralories: both showed weaker effects on tuberculin response than liquid $\mathrm{BCG}$.

Key words: BCG. Freezedried preparation. Field Study. Liophilized versus liquid BCG. Quality Control. Tubercu . lin responsc).
\end{abstract}

La Tuberculosis (TBC) continúa siendo un importante problema de salud pública en los países en vía de desarrollo.

La morbilidad por TBC es aún alta en Chile: 6.941 casos nuevos fueton notificados en 1982, a pesar del descenso de $21 \%$ experimentado por su Tasa, que de 76,9 por $100.000 \mathrm{hb}$. en 1978 , bajó a 60.4 en $1982^{1}$. La morbilidad se concentra en los mayores de 15 años, con $93,9 \%$ de los casus: sin embargo, en 1982 , se produjo un número absoluto no despreciable de 420 casos notifica. dos en menores de 15 años.

La mortalidad por TBC ha bajado en $47.4 \%$ en los últimos 5 años, observándose una Tasa de Mortalidad de 8.1 en 1983. Dentro de las enfermedades infecciosas transmisibles, la TBC es responsable de $72 \%$ de las muertes ${ }^{2}$. lo que acentua la importancia de afirmar los mecanismos de control de la enfermedad.

En 1920 el Dr. Fontecilla introdujo en Chile la vacuna $\mathrm{BCG}^{3}$ la que protege, aproximadamente, a $78 \%$ de los vacunados ${ }^{4}$. Actualmente se ha alcanzado una cobertura de $91 \%$ de vacunación en los Recién Nacidos del pais, y de $83,1 \%$ en la revacunación de escolares de primer año básico²

1. Químico Farmacécutico, Instituto de Salud Pública de Chile.

2. Pudiatra Docente. Iscuela Salud Pública, Universidad de Chile.

3. Enfermera Docenté, Carrera de Infermería, Universidad de Chile.

4. Matrona Maternidad Hospital del Salvador, S.S.M Oriente.
El Instituto de Salud Pública de Chile, proporcionó hasta 1982 la vacuna BCG líquida que se emplea en el país en recién nacidos ${ }^{5}$. Posteriormente se introdujo el uso de vacunas $\mathrm{BCG}$ liofilizadas, una de las cuales (Connaught) fue objeto de un estudio de campo realizado por este mismo grupo ${ }^{6}$. Siguiendo recomendaciones de la $\mathrm{OMS}^{7}$, presentamos esta investigación realizada con otra vacuna BCG liofilizada, del Instituto Rhodia Merieux, Francia, con el propósito de contribuir al conocimiento de algunos aspectos de ella en su aplicación en terreno y compararla con la vacuna liofilizada de Laboratorios Connaught y la vacuna BCG liquida producida en Chile ${ }^{6-8}$

Los objetivos del estudio fueron determinar la presencia o ausencia de cicatriz BCG producida por la vacuna del lnstituto Rhodia Merieux y compararla con la cicatriz producida por la vacuna BCG de Laboratorio Connaught; conocer la media del tamaño de la reacción tuberculínica provocada por la vacuna en estudio y compararla con la encontrada después de usar vacunas de Laboratorios Connaught y BCG líquida del antiguo Instituto Bacteriológico de Chile empleada antes de Octubre de 1982; realizar el control de calidad del lote de vacuna liofilizada del Instituto Rhodia Merieux que se empleó en esta investigación.

\section{MATERIAL Y METODO}

El estudio se efectuó en la Maternidad del Hospital del Salvador, Servicio de Salud Metropo- 
litano Oriente (SSM.OR), en nir̆os nacidos entre el 28 de Mayo y 13 de Julio de 1984. Se estudiaron 116 niños captados en el período de recién nacido, que fueron vacunados por la matrona participante. La madré de cada niño vacunado fue citada por escrito, especificando fecha $y$ lugar de control a los 4 meses de edad, en el Consultorio Santa Julia, SSM.OR. Se reforzó la citdción 15 días antes de la fecha con una carta al domicilio recordando el lugar $y$ hora de control.

La vacunación se realizó mediante inyección intradérmica, con jeringa Omega, de 0,1 $\mathrm{ml}$. de la vacuna $B C G$ liofilizada del Instituto Rhodia Merieux, Francia, preparada con una cepa tipo Londres Fi0 y presentada en frasco-ampollas incoloros con 20 dosis.

El control se realizó en dos etapas; en la primera se efectuó la prueba tuberculínica, la lectura de la cicatriz BCG y se buscó la existencia de reacción ganglionar local. En la segunda, 72 horas después, efectuó la lectura de la reacción tuberculínica, en su diámetro transverso, una persona experta en técnica y lectura de PPD.

Para la prueba tuberculínica se usó el PPD Rt23 con Tween 80 del Instituto Estatal del Suero de Copenhagen, diluído por el Instituto de Salud Pública de Chile. Se utilizó la via intradérmica colocando $0.1 \mathrm{ml}$. equivalente a 2 Unidades de Tuberculina, en el tercio medio del antebrazo izquierdo.

E1 control de calidad con respecto al contenido de partículas cultivables de la vacuna, se efectuó en el Departamento de Control Nacional del Instituto de Salud Pública de Chile. Se analizaron 2 muestras de vacunas retiradas del centro de vacunación de la Maternidad del Salvador, al comienzo y al final del período de estudio y además una muestra mantenida en refrigetación en el Laboratorio de Control Nacional.

Se debe señalar que la técniça empleada en relación a la colocación del BCG y PPD y la lectura del PPD, como asimismo los procedimientos para el control de calidad, fueron los mismos empleados al estudiar la vacuna BCG liofilizada de Laboratorios Connaught ${ }^{6}$.

\section{RESULTADOS}

En la Tabla 1 se presentan los resultados del tamaño de la cicatriz BCG, el $33,6 \%$ de los niños tuvieron cicatriz 4 a $5 \mathrm{~mm}$, el promedio fue de $4,6 \mathrm{~mm}$. con una D.S. de 2,4. Si comparamos estos resultados con la cicatriz obtenida con la vacuna BCG Connaught, se puede apreciar que son similares (Tabla 2).

En la Tabla 3 se muestran los resultados de la reacción tuberculínica, el $36 \%$ de los niños tuvieron un PPD entre 4 y $7 \mathrm{~mm}$., el promedio obtenido fue de $4.8 \mathrm{~mm}$, con und D.S. de 3,5 . Esta ha sido la respuesta PPD más débil observada por los autores.

Tabla 1.

Tamaño de cicatriz en nínos de 4 meses Vacuna BCG Liofilizada Rhodia Merjeux S.S.M. Oriente, Suntiago 1984.

\begin{tabular}{ccc}
\hline $\begin{array}{c}\text { Cicatriz. BCG } \\
\text { mm. }\end{array}$ & No & $\%$ \\
\hline $0-1$ & 8 & 6.9 \\
$2-3$ & 32 & 27.6 \\
$4-5$ & 39 & 33.6 \\
$6-7$ & 23 & 19.8 \\
$8 \cdot 9$ & 10 & 8.6 \\
$10 \quad 11$ & 4 & 3.5 \\
\hline Total & 116 & 100.0 \\
\hline
\end{tabular}

Media $4.6 \quad$ D.S. $2.4 \mathrm{~mm}$.

Tabla 2.

Tamaño medio de la cieatriz con 2 vacunas BCG Liofilizadas

\begin{tabular}{lcc}
\hline Tipo de Vacuna BCG & $\begin{array}{c}\text { D. Standard Tamaño cicatriz } \\
\text { en mm. }\end{array}$ & promedio en mrn. \\
\hline Liofilizada L. Connaught & 2.13 & 5.27 \\
Liotilizada L. Merieux & 2.36 & 4.62 \\
\hline
\end{tabular}

Tabla 3 .

Reacción tuberculínica en niños de 4 meses Vacuna Liofilizada Rhodia Merieux S.S.M. Oriente, Santiago 1984

\begin{tabular}{ccc}
\hline PPD mm. & No & $\%$ \\
\hline $0-1$ & 28 & 26.7 \\
23 & 13 & 12.5 \\
$4-5$ & 18 & 17.1 \\
$6-7$ & 18 & 17.1 \\
$8-9$ & 16 & 15.2 \\
$10-11$ & 10 & 9.5 \\
$12-13$ & 2 & 1.9 \\
- Total & 105 & 100.0 \\
\hline
\end{tabular}

Media $4.8 \mathrm{~mm}$. D.S. $3.5 \mathrm{~mm}$.

El comparar la reacción tuberculínica obtenida con ta vacuna BCG líquida del Instituto Bacteriológico de Chile y las vacunas liofilizadas Connaught y Merieux encontramos mayor respuesta con vacuna BCG liquida (Tabla 4). La aplicación del método estadístico de la varianza para comparar promedios ${ }^{9-1} 0$ permitió concluir que habia diferencias significativas entre los valores de reacción tuberculínica observados 
( $p<0.001)$. Estas diferencias se establecen al comparar la vacuna líquida con cada una de las liofilizadas entre sí.

\section{Tablo 4.}

Tamatio de PPD en niños de 4 meses con 3 Vacunas $B C G$ S.S.M. Oriente, Santiago.

\begin{tabular}{|c|c|c|c|}
\hline Tipo de Vacuna BCG & $\begin{array}{c}\text { Tamaño PPD } \\
\text { men. }\end{array}$ & $\begin{array}{l} \pm \text { D.S. } \\
\mathrm{mm} \text {. }\end{array}$ & $\begin{array}{l}\text { Sujrtos } \\
\text { iN\} }\end{array}$ \\
\hline \multicolumn{4}{|l|}{ Liquida Inst. } \\
\hline Battersolog. Chile & 11.1 & 5.5 & 76 \\
\hline Liofilizads Connaught & 5.5 & 3.5 & 74 \\
\hline Liofilizadid Mericur & 4.8 & 3.5 & 105 \\
\hline
\end{tabular}

$\mathbf{I}=71,95$

F crit. $=3,00 \mathrm{p}<0.001$

En la Tabla 5 se expresa la calidad de la vacuna "in vitro" según el contenido de partículas cultivables ${ }^{1}$. La vacuna estudiada denostró buena estabilidad durante el periodo de observación. Las variaciones observadas son las propias de las determinaciones biológicas.

\section{COMENTARIOS Y CONCLUSIONES}

Motivados por la introducción de una vacuna BCG liofilizada de un laboratorio diferente $y$ por el hecho de haber realizado ya otras evaluaciones de Vacuna BCG. pareció interesante estudiar en terreno esta vacuia y compararla con otras em. pleadas en Chile. El hecho de haber utilizado el mismo método y PPD, permite comparar las vacunas controlando las variables que intervienen en el proceso de respuesta.

No encontramos diferencias apreciables en el tamaño de la cicatriz ubtenida, al comparar ésta, con la vacuna BCG liofilizada Connaught, y otros tipos de vacuna utilizados en Chile y en el extranjero ${ }^{12-13}$ señalando, como lo hicimos antes, que no parece haber correlación entre el tamaño de la cicatriz y la protección conferida por la vacuna: hay experiencias que demuestran que un alto contenido de gérmenes muertos puede dar buenas cicatrices sin buena reacción tuberculinica 14

Las respuestas de la reacción tuberculínica provocada por las vacunas Merieux y Connaught son bastantes similares y en ambos casos menores que las obtenidas con la liquida. Si consideramos que una buena manera de evaluar la calidad de la vacuna es esta respuesta, podríamos decir que las primeras son considerablemente más débiles que la última y semejantes entre si.

Debemos considerar que al estimar, por medio del PPD. la respuesta inmunitaria inducida por la vacuna estamos laciéndolo indirectamente. En Chile es conocida la baja respuesta al PPD en comparación con otros paises ${ }^{15}$ y no creemos que li población infantil de 0 a $4 \mathrm{~m}$. hubjese cambiado tanto entre 1976 y 1984 como para explicar diferentes respuestas al PPD por variaciones del huésped. De este modo pademos concluir, que por razones hasta ahora desconocidas las vacunas BCG liofilizadas estudiadas inducen reacciones tuberculínicas más pobres que la vacuna líquida aplicada en Chile hasta 1982. Lo anterior podría significar que estas vacunas otorgan menor protección que otras empleadas con anterioridad en Chile.

No ocurre lo mismo al estudiar la calidad de la vacuna BCG Merieux "in vitro". Los controles indican que es estable si se mantiene en buenas condiciones en la Cadena de Frío y promisoria desde el punto de vista operacional, a pesar del frasco incoloro y de falias ocasionales de vacío.

\section{RESUMEN}

Se realiza un estudio de campo con 116 niños de 4 meses, vacunados al nacer con la vacuna

Tabla 5.

Control de calidad de las muestras de Vacuna BCG I.iofilizada Rhodia Merieux*

\begin{tabular}{clcc}
\hline Muestra No & Origen & Fecha & \multicolumn{1}{c}{$\begin{array}{c}\text { Resuitidos (particulas } \\
\text { cultivables mill/m!.) }\end{array}$} \\
\hline 1 & $\begin{array}{l}\text { Centrit Abastecimiento } \\
\text { Control de Serie }\end{array}$ & Enero 1984 \\
2 & $\begin{array}{l}\text { Maternidad del Salvador } \\
\text { Laboratorio Control Nacional }\end{array}$ & Junio 1984 & 7.6 \\
3 & Junio 1984 & 9.8 & 7.8 \\
\hline
\end{tabular}

(*) Realizados de acuerdo a Guía Técnica 77.9 de la OMS, utjlizando el medio de Lowenste in Jensen ${ }^{11}$. 
BCG Liofilizada del Instituto Rhodia Merieux, importada de Francia y sometida a control de calidad por el Instituto de Salud Pública de Chile. En el presente trabajo se empleó el mismo método $y$ observadores que en un trabajo anterior sobre la vacuna BCG Liofilizada de Laboratorios Connaught (Canadá).

La vacuna en estudio deja una cicatriz seme. jante a las utilizadas anteriormente en Chile; la respuesta Tuberculínica es débil: media $4.8 \mathrm{~mm}$. D.S. $3.5 \mathrm{~mm}$., y se encontró una diferencia significativa al compararla con la vacuna BCG Liquida fabricada por el Instituto de Salud Pública de Chile la que aparece dando una mucho mejor respuesta tuberculinica ( $\mathrm{p}=<0.001)$.

Al comparar las dos vacunas BCG Liofilizadas, no se advierten diferencias importantes. demostrándose ambas considerablemente más débiles en su respuesta tuberculínica que la vacuna BCG Liquida.

Los controles de calidad "in vitro" de la vacuna BCG Liofilizada del Instituto Rhodia Merieux demastraron que es estable y promisoria desde el punto de vista operacional.

\section{REFERENCIAS}

1. Ministerio de Salted Departamento de Planificación: Enfermcdades de Notificación Obligatoria. Anuario 1982-1983.

2. Toro J.: Epidemiologia de la Tuberculosis en Chile. Bol. Vigil. Epidemiol. 11:3, 1984.

3. Araya, Ch. P.: Historia de la vacunación antituberculosa con BOG en el ser humano en Chile. Bol. Médico--Social No 164 a $168,1948$.
4. Anónimo: Fourth report. Br. Med. Research Council Bull WHO 46: 371, 1972.

5. Anómimo: Uso de Vacuna BCG liofilizada para Recién Nacidos Rep. de Chile, Mínistetío de Salud, Depto. de apoyo a los programas, circular 117, Julio 1982.

6. Azzira, I, Carrasco R. y cols.: Reacción Tuberculínica y Cicatriz $\mathrm{BCG}_{\mathrm{s}}$ en lactantes vacunados con BCG liofilizado. Rev. Chil. Pediatr. 55: 313, 1984.

7. Anónimo: Comité de lixpertos de la OMS en Patrones Biológicos. $30^{\circ}$ Informe. Ser. Inf. Tec. OMS 638: 156, 1979 .

8. Rojas, L., Azzini, J., Ferrer, $x$. y cols.: Vacunación BCG en recién nacidos con vacuna preparada de cultivos de 10 y 14 días $2 a s$ Jornadas de Microbiología U.C.V. Octubre 1976.

9. Zuwaylif, H.: Estadistica Gencral Aplicada. Fondo Educativo Interamericano. 1977.

10. Anónimo: The American Statistician 35; 136, 1931 .

1. Anónimo: "In vitro" essays of BCG products. WHO TB Tech. Guide 9: 3, 1977.

12. Ferrer, X., Latrach, C. y cols.: Piesenila de cicatriz BCG y reaccion tuberculínicu en esculares de 10 Año Básico, S.S.M. Onente. Bol. Vigil. Fpidemiol. Ministerio de Silud, Chile 10: 7, 1983.

13. Edwards, L.B., Gelting, A.S.: Fiffects of age of vaccine and variation in storage temperature and dosage on allergy production und vaccination lessions 10 weeksi after vaccination. Bull WHO 3 : $279,1950$.

14. Nissen Mayer $S_{\text {, }}$ Palmer C.E.: Field Studies of the significance ot dead Bacilli in BCG vaceinc. Bull WHO 7: 201, 1952 .

15. Borleselle $O$., Rojas I..: Consideraciones nobre 6.045 pruebas tuberculínicas en Santiago. Estudio cooperativo del Comité de lucha Anti-TBC de la $V$ Zona de salud SNS SERMLNA Material impreso. Campaña Anti-TBC. Servicio de Divulgación $1969-1970$. 